\title{
Differential expression of stem cell-like proteins in normal, hyperplastic and dysplastic oral epithelium
}

\author{
Sarah Mohammed Mohammed BARAKAT, Chong Huat SIAR
}

Department of Oro-Maxillofacial Surgical and Medical Sciences, Faculty of Dentistry, University of Malaya, Kuala Lumpur, Malaysia.

Corresponding address: Chong-Huat Siar - Department of Oro-Maxillofacial Surgical and Medical Sciences - Faculty of Dentistry - University of Malaya 50603 Kuala Lumpur - Malaysia - Tel: +603-7967-4859 - Fax: 603-7967-4531 - e-mail: siarch@um.edu.my

Submitted: July 16, 2014 - Modification: October 23, 2014 - Accepted: December 1, 2014

\section{ABSTRACT}

\begin{abstract}
bjective: The identification of stem cells (SC) remains challenging. In the human oral mucosal epithelium, these cells are believed to be in the basal layer (stem cell niche), but their exact location is unclear. The aim of this study was to examine the dysplastic oral epithelium for these SC-like proteins in order to assess their diagnostic value as biomarkers complementing the histological grading of dysplasia. Material and Methods: Thirty oral epithelial dysplasia (OED), 25 oral lichen planus (OLP), 10 oral hyperkeratosis and 5 normal oral epithelium (OE) were immunohistochemically examined for four SC markers [integrin $\beta 1$, neuron-glial-2 (NG2), notch 1 (N1) and keratin 15 (K15)]. Results: Three of four SC markers were heterogeneously detected in all samples. K15 overexpression in the lower two-thirds of severe OED suggests an expanded SC niche. Integrin $\beta 1$ distribution pattern was not measurably different between OEDs and control. NG2 was almost negative to absent in all samples examined. N1 expression was weak and highly variable in normal and dysplastic epithelium, making it an unreliable epithelial stem cell marker. Conclusions: Present findings suggest that these markers were unable to identify individual epithelial stem cells. Instead, subpopulations of cells, most probably stem cells and transit amplifying cells with stem cell-like properties were identified in the dysplastic oral epithelium. The characteristic expressions of K15 might be of diagnostic value for oral dysplasia and should be investigated further.
\end{abstract}

Keywords: Stem cells. Integrin $\beta 1$. Notch 1. Keratin-15. Dysplasia.

\section{INTRODUCTION}

Stem cells constitute a distinct subset of cells characterized by their capacity to self-renewal and differentiation into multi-lineage cellular constituents of a specific tissue or organ ${ }^{25}$. Although the phenotypic characteristics of these cells are not fully realized, their morphological features include small size, poor differentiation and primitive cytoplasm. These unique cells may undergo either symmetrical cell division, yielding two identical daughter stem cells, or asymmetrical cell division, to give rise to one daughter stem cell that replaces the original mother stem cell and a transit amplifying cell (TAC), which enters limited rounds of rapid cell division that increase their number, thereby preserving tissue integrity ${ }^{22}$. Stem cells are known to reside in specific locations known as stem cell niches ${ }^{12,22}$. In the human oral mucosal epithelium, stem cells are believed to be in the basal layer, but their exact localization has not been fully elucidated ${ }^{11}$.

Oral potentially malignant disorder (OPMD) is the term recommended by the World Health Organization Collaborating Center for Oral Cancer and Precancer Working Group over oral premalignant lesions and conditions as it indicates that not all lesions describe under this term may transform to cancer ${ }^{1,33}$. The most common OPMDs are leukoplakia, erythroplakia and oral lichen planus (OLP). Although the degree of epithelial dysplasia is generally used for assessing the risk of malignant transformation in OPMDs, 
currently it is impossible to predict accurately which lesions would progress to malignancy ${ }^{30}$. For OLP, studies have reported varying rates of malignant transformation ranging from $0.5 \%$ up to $2.9 \%{ }^{3}$. The role that stem cells play in the evolution of OPMDs and their progression is unclear.

The identification of stem cells remains challenging as they account for less than $1 \%$ of the adult cell population and their capacity to divide is diminished once they are removed from the body ${ }^{16}$. There are several ways to detect stem cells whether in vivo or in vitro. Stem cells are characterized by specific markers such as antigens, molecules and signaling pathways. A reliable marker is necessary to characterize these cells, and putative stem cell markers need to be assessed for their differential specificities in identifying this cellular subset. On the other hand, it is still unclear whether every markerpositive cell has the property of a stem cell. In human epidermis, surrogate markers including keratins 15 and 19 , integrins $\alpha 6$ and $\beta 1$, and p63 are highly expressed by the epidermal stem cells and early progenitor cells. These markers purportedly identify stem cell zone if not individual stem cells $7,24,28$. Whilst stem cells have been widely investigated at tissue sites including the skin $7,14,15,21,24,27$, central nervous system ${ }^{9}$ and cartilaginous tissues ${ }^{10}$, but much less is known about their characteristics in the oral epithelium ${ }^{13,17}$. The aim of this study was to determine the differential expression of four stem cell markers, namely integrin beta 1 ( $\beta 1)$, neuron-glial-2 (NG2), keratin 15 (K15) and notch 1 (N1) in the three constitutive tissue layers of dysplastic oral epithelium and compare their expression patterns with those of oral lichen planus (OLP), oral hyperkeratosis (OHK) and normal oral mucosa (NOM). The justification for choosing these four markers are: i) integrin $\beta 1$, a component of integrin complexes, is expressed in high levels by epithelial stem cells ${ }^{13,14}$, ii) NG2, a chondroitin sulfate proteoglycan, is expressed by stem cells associated with interfollicular epidermis and hair follicles ${ }^{15}$, iii) N1 signaling is a direct determinant of stem cell fate in keratinocytes ${ }^{27}$, and iv) $\mathrm{K} 15$, an intermediate filament protein is expressed in hair follicle bulge stem cells ${ }^{20}$.

\section{MATERIAL AND METHODS}

\section{Samples}

The sample consisted of formalin-fixed paraffinembedded blocks of 30 cases of oral epithelial dysplasia (OED) [10 mild (Mi), 10 moderate (Mo), and 10 severe (Se)] 25 OLP and $10 \mathrm{OHK}$, retrieved from the archival records of the department of oral pathology. All these cases were reviewed by a qualified oral pathologist and selected according to the diagnostic criteria of the $\mathrm{WHO}^{1,33}$. Five cases of normal oral mucosa (NOM) were used as a control group.

\section{Immunohistochemistry}

Four commercially available antibodies, namely rabbit polyclonal antibody to integrin $\beta 1$ (ab52971), notch 1 (ab27526) and keratin 15 (ab53116) (Abcam Inc., Cambridge, MA, USA), and rabbit monoclonal [LHM2] to NG2 (ab20156) (Abcam Inc., Cambridge, MA, USA) were obtained.

For immunohistochemistry, $4 \mu \mathrm{m}$-thick sections were prepared from the formalin-fixed paraffinembedded blocks and mounted on salinized slides. These sections were deparaffinized, rehydrated, immersed in citrate buffer $(\mathrm{pH} 6)$, heated in a scientific microwave oven (H2850 Microwave Processor, EB Sciences Inc., East Granby, CT, USA) for $10 \mathrm{~min}$ at $99^{\circ} \mathrm{C}$ for antigen retrieval, and were allowed to cool down at room temperature before blockade of endogenous peroxidase with $3 \%$ hydrogen peroxide in methanol for $10 \mathrm{~min}$. The sections were then incubated with the optimally diluted primary antibodies [integrin $\beta 1$ (1:100), NG2 (1:400), N1 (1:200) and K15 (1:200)] for 30 min at room temperature. An Envision kit (Dako REAL Envision Detection System, Peroxidase/ $\mathrm{DAB}+$ Rabbit/Mouse, Dako Corporation, Carpinteria, CA, USA) was used for the incubation with the secondary antibody for 1 hour, then the peroxidase activity was allowed to develop by incubating the sections in diaminobenzidine (DAB) for $5 \mathrm{~min}$. Sections were then counterstained with Harris's haematoxylin. Positive and negative controls were used according to manufacturer's instructions.

\section{Immunohistochemical analysis}

For assessment of immunoexpression, a semiquantitative method was performed by two operators who were calibrated. Immunoreactivity was categorized as negative $(-)$ or positive $(+)$. Positive staining was assigned when cytoplasmic, membranous or nuclear immunoreactivity could be identified in any number of cells. For each positive case, five fields ("hot spots") were chosen according to the areas showing the most intense staining at 200x magnification. In each field, staining was evaluated according to the specific epithelial layers: stratum basale, spinosum or corneum. The intensity of staining was categorized as mild $(+)$ when the stain was present in the focal areas $(<25 \%)$; moderate $(++)$ when the staining was evident in significant areas (25\%-50\%); and strong $(+++)$ when the stain was present in predominant areas $(>50 \%)$. Their mean percentage scores were obtained. Localization of staining was also categorized as cytoplasmic, membranous and/ or nuclear. Representative digital images were captured using the Olyvia DotSlide Virtual Slide 
System (Olympus Imaging Inc., Tokyo, Japan).

\section{RESULTS}

The immunohistochemical results and distribution of cases (NOM, OHK, OLP, MiOED, MoOED and SeOED) according to their mean percentage scores for the four stem cell markers (integrin $\beta 1, N G 2, N 1$ and $\mathrm{K} 15$ ) in the three constitutive epithelial layers are detailed in Table 1 and illustrated in Figures 1-4.

\section{Integrin $\beta 1$}

Integrin $\beta 1$ was detected in nearly all cases of OLP, OHK, OED and NOM. (Table 1). Their distribution patterns were not measurably different between the test and control samples. Integrin $\beta 1$ protein localization was cytoplasmic and nuclear. Positive expression for this protein was largely observed in the basal layer of the oral epithelium and to a lesser extent in the suprabasal keratinocytes (Figures 1A-F). Two OED samples (1MoOED and 1 SeOED,
$10 \%$ of total) demonstrated moderate signaling for this biomarker in the spinous layer (Figures 1E, F) (Table 1).

\section{NG2}

NG2 was predominantly non-detectable in both test and control samples (Table 1). In those cases that expressed NG2 positivity, staining was generally weak and localized in the membrane and cytoplasm. Their distribution patterns and/or lack of immunoexpression are illustrated in Figures 2A-F.

\section{Notch 1}

N1 signal was weak and highly variable. Weak basal signal was detected in normal epithelium while a stronger basal-suprabasal signal was observed in moderate dysplastic tissue. In those cases that expressed N1 positivity, protein localization was predominantly cytoplasmic and membranous. Their patterns of distribution in the various epithelial layers were heterogeneous and not measurably

Table 1- Distribution of stem cell-like protein markers in normal oral mucosa (NOM), oral hyperkeratosis (OHK), oral lichen planus (OLP) and oral epithelial dysplasia (OED)

\begin{tabular}{|c|c|c|c|c|c|c|c|c|c|c|c|c|c|c|c|c|}
\hline \multirow{3}{*}{ Variables } & \multicolumn{16}{|c|}{ Staining expression levels of stem cell-like protein markers (\%) } \\
\hline & \multicolumn{4}{|c|}{ Integrin $\beta 1$} & \multicolumn{4}{|c|}{ Neuron-glia- 2} & \multicolumn{4}{|c|}{ Notch 1} & \multicolumn{4}{|c|}{ Keratin 15} \\
\hline & - & + & ++ & +++ & - & + & ++ & +++ & - & + & ++ & +++ & - & + & ++ & +++ \\
\hline \multicolumn{17}{|l|}{ NOM $(n=5)$} \\
\hline S. corneum & $4(80)$ & $1(20)$ & 0 & 0 & $4(80)$ & $1(20)$ & 0 & 0 & 0 & 0 & $1(20)$ & $4(80)$ & 0 & $2(40)$ & $1(20)$ & $2(40)$ \\
\hline S. spinosum & $4(80)$ & $1(20)$ & 0 & 0 & $4(80)$ & $1(20)$ & 0 & 0 & 0 & 0 & 0 & $5(100)$ & $2(40)$ & $1(20)$ & 0 & $2(40)$ \\
\hline S. basale & 0 & 0 & $4(80)$ & $1(20)$ & $4(80)$ & $1(20)$ & 0 & 0 & $3(60)$ & 0 & $2(40)$ & 0 & 0 & $1(20)$ & 0 & $4(80)$ \\
\hline \multicolumn{17}{|l|}{ OHK $(n=10)$} \\
\hline S. corneum & $9(90)$ & $1(10)$ & 0 & 0 & $8(80)$ & $2(20)$ & 0 & 0 & 0 & $1(10)$ & 0 & $9(90)$ & $2(20)$ & $5(50)$ & $3(30)$ & 0 \\
\hline S. spinosum & $9(90)$ & $1(10)$ & 0 & 0 & $9(90)$ & $1(10)$ & 0 & 0 & 0 & $3(30)$ & $1(10)$ & $6(60)$ & $3(30)$ & $1(10)$ & $1(10)$ & $5(50)$ \\
\hline S. basale & 0 & 0 & $8(80)$ & $2(20)$ & $9(90)$ & $1(10)$ & 0 & 0 & 0 & $4(40)$ & $1(10)$ & $5(50)$ & 0 & $4(40)$ & $1(10)$ & $5(50)$ \\
\hline \multicolumn{17}{|l|}{ OLP $(n=25)$} \\
\hline S. corneum & $25(100)$ & 0 & 0 & 0 & $20(80)$ & $4(16)$ & 0 & $1(4)$ & 0 & $4(16)$ & $7(28)$ & $14(56)$ & $10(40)$ & $10(40)$ & $5(20)$ & 0 \\
\hline S. spinosum & $20(80)$ & $5(20)$ & 0 & 0 & 23(92) & $1(4)$ & $1(4)$ & 0 & $1(4)$ & $3(12)$ & $3(12)$ & $18(72)$ & $5(20)$ & $11(44)$ & $6(24)$ & $3(12)$ \\
\hline S. basale & $1(4)$ & $1(4)$ & $18(72)$ & $5(20)$ & 23(92) & $2(8)$ & 0 & 0 & $13(52)$ & $3(12)$ & $6(24)$ & $3(12)$ & $4(16)$ & $5(20)$ & $3(12)$ & $13(52)$ \\
\hline \multicolumn{17}{|l|}{$\operatorname{MiOED}(n=10)$} \\
\hline S. corneum & $9(90)$ & $1(10)$ & 0 & 0 & $6(60)$ & $3(30)$ & $1(10)$ & 0 & $1(10)$ & 0 & $2(20)$ & $7(70)$ & $2(20)$ & $2(20)$ & $6(60)$ & 0 \\
\hline S. spinosum & $9(90)$ & $1(10)$ & 0 & 0 & $6(60)$ & $4(40)$ & 0 & 0 & $1(10)$ & 0 & $3(30)$ & $6(60)$ & 0 & $4(40)$ & $2(20)$ & $4(40)$ \\
\hline S. basale & 0 & $1(10)$ & $4(40)$ & $5(50)$ & $7(70)$ & $3(30)$ & 0 & 0 & $7(70)$ & $1(10)$ & $2(20)$ & 0) & 0 & $1(10)$ & $2(20)$ & $7(70)$ \\
\hline \multicolumn{17}{|l|}{ MoOED $(n=10)$} \\
\hline S. corneum & $9(90)$ & $1(10)$ & 0 & 0 & $7(70)$ & $3(30)$ & 0 & 0 & $1(10)$ & $1(10)$ & $1(10)$ & $7(70)$ & $1(10)$ & 0 & $2(20)$ & $7(70)$ \\
\hline S. spinosum & $9(90)$ & 0 & $1(10)$ & 0 & $7(70)$ & $1(10)$ & $1(10)$ & $1(10)$ & $3(30)$ & $2(20)$ & $1(10)$ & $4(40)$ & 0 & $3(30)$ & $4(40)$ & $3(30)$ \\
\hline S. basale & 0 & $1(10)$ & $4(40)$ & $5(50)$ & $10(100)$ & 0 & 0 & 0 & $8(80)$ & $2(20)$ & 0 & 0 & $3(30)$ & $4(40)$ & 0 & $3(30)$ \\
\hline \multicolumn{17}{|l|}{ SeOED $(n=10)$} \\
\hline S. corneum & $9(90)$ & $1(10)$ & 0 & 0 & $9(90)$ & $1(10)$ & 0 & 0 & $2(20)$ & $3(30)$ & $2(20)$ & $3(30)$ & $1(10)$ & $8(80)$ & 0 & $1(10)$ \\
\hline S. spinosum & $9(90)$ & 0 & $1(10)$ & 0 & $10(100)$ & 0 & 0 & 0 & $6(60)$ & $4(40)$ & 0 & 0 & $1(10)$ & $4(40)$ & $1(10)$ & $4(40)$ \\
\hline S. basale & $1(10)$ & $1(10)$ & $4(40)$ & $4(40)$ & $10(100)$ & 0 & 0 & 0 & $10(100)$ & 0 & 0 & 0 & $1(10)$ & $1(10)$ & $2(20)$ & $6(60)$ \\
\hline
\end{tabular}

$\mathrm{Mi}=$ mild; $\mathrm{Mo}=$ moderate; $\mathrm{Se}=$ severe; $\mathrm{S}=$ Stratum; - = negative staining; + = mild staining; ++ = moderate staining; +++ $=$ strong staining. 

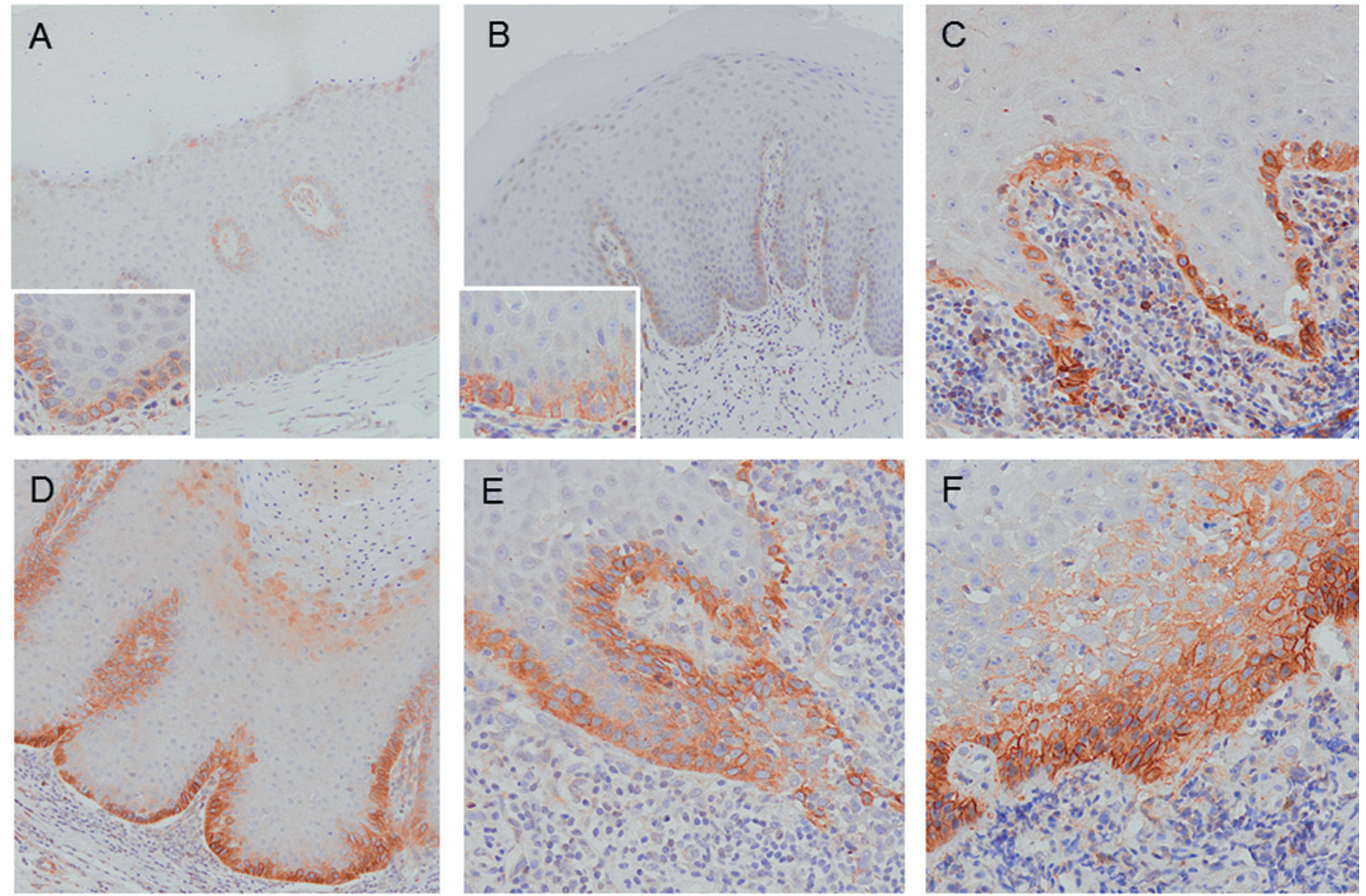

Figure 1- Integrin $\beta 1$ distribution patterns in the various epithelial layers of normal oral mucosa (A), oral hyperkeratosis (B), oral lichen planus $(C)$, mild oral epithelial dysplasia $(D)$, moderate oral epithelial dysplasia $(E)$ and severe oral epithelial dysplasia (F). Original magnification 100x (A, B); 200x (Inset A, Inset B, C, D, E, F)
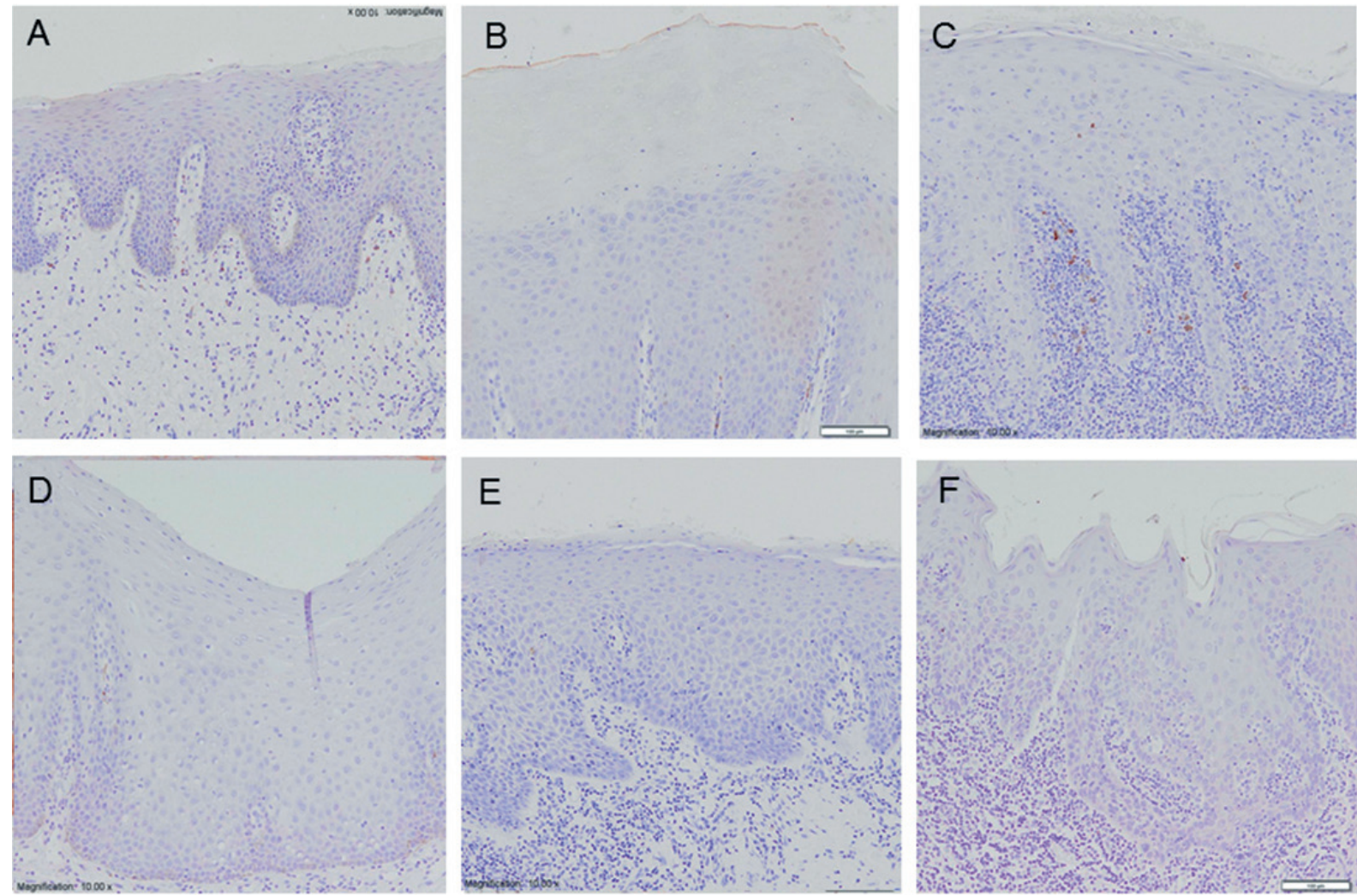

Figure 2- Neuron-glia-2 (NG2) distribution patterns in the various epithelial layers of normal oral mucosa (A), oral hyperkeratosis $(B)$, oral lichen planus $(C)$, mild oral epithelial dysplasia $(D)$, moderate oral epithelial dysplasia (E) and severe oral epithelial dysplasia $(F)$. Original magnification 100x (A, B, C, D, E, F) 

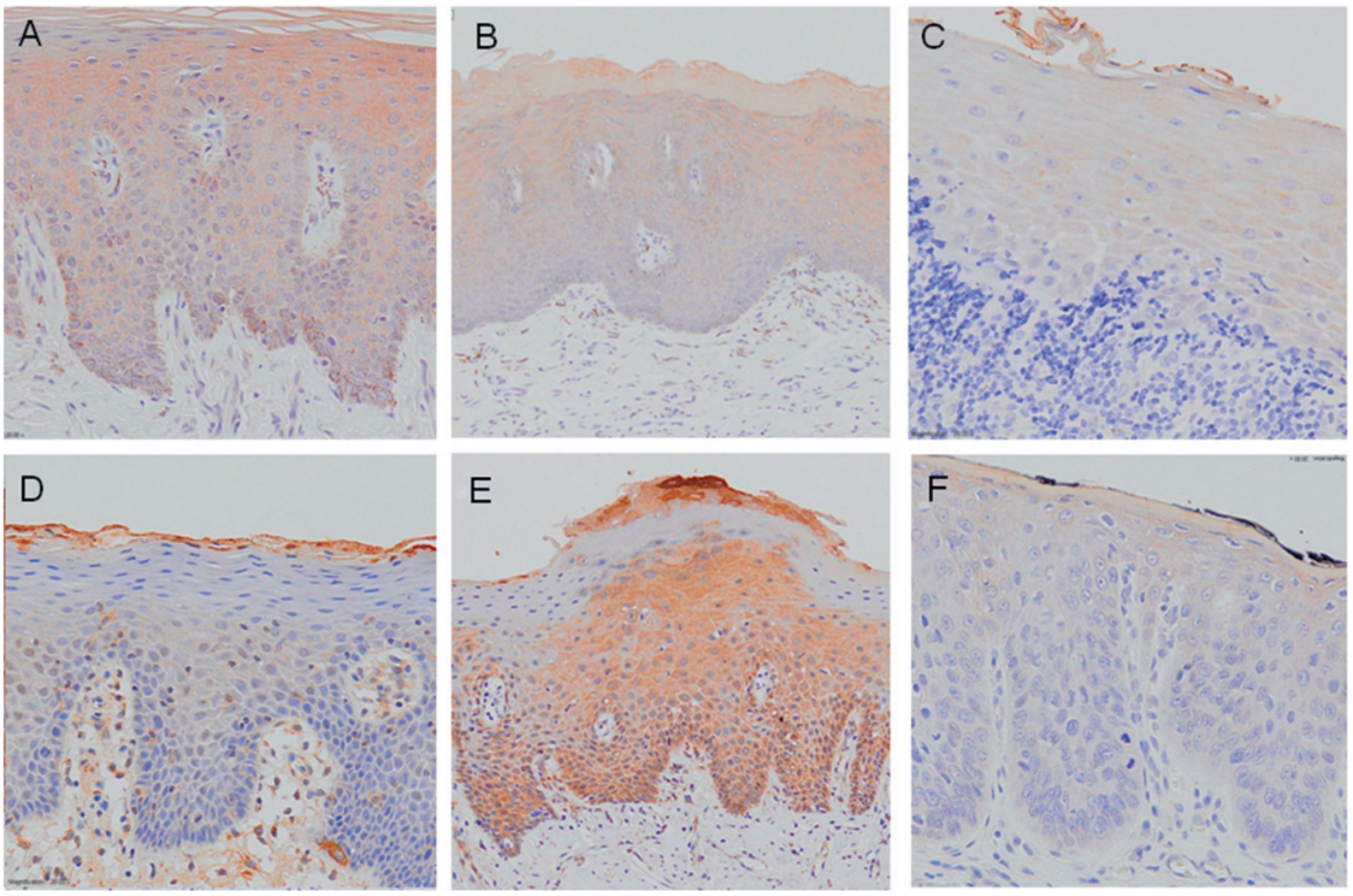

Figure 3- Notch 1 (N1) distribution patterns in the various epithelial layers of normal oral mucosa (A), oral hyperkeratosis $(B)$, oral lichen planus $(C)$, mild oral epithelial dysplasia (D), moderate oral epithelial dysplasia $(E)$ and severe oral epithelial dysplasia (F). Original magnification 100x (A, B, D); 200x (C, E, F)
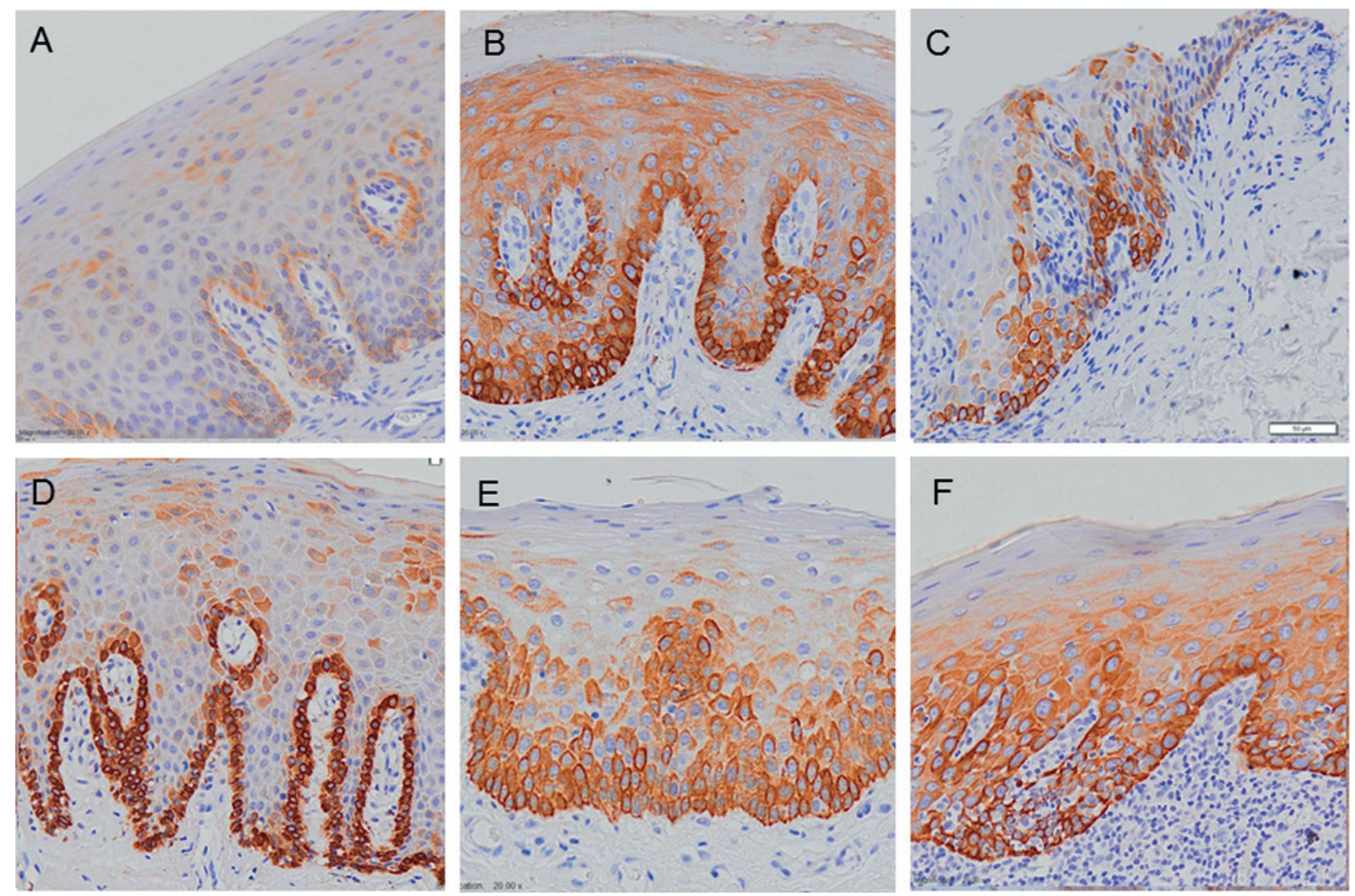

Figure 4- Keratin 15 (K15) distribution patterns in the various epithelial layers of normal oral mucosa (A), oral hyperkeratosis $(B)$, oral lichen planus $(C)$, mild oral epithelial dysplasia (D), moderate oral epithelial dysplasia $(E)$ and severe oral epithelial dysplasia (F). Original magnification 100x (B, E, E Inset); 200x (A, C, D, F) 
different between test and control (Figures $3 A-F$ ). Nuclear immunopositivity was most apparent in SeOED (Figure 4F).

\section{Keratin 15}

K15 was not detected in $4(16 \%)$ cases of OLP, $3(30 \%)$ MoOED and 1 case $(10 \%)$ of SeOED (Table 1). In those cases that were positive for $\mathrm{K} 15$, protein localization was membranous and cytoplasmic. Their distribution patterns are shown in Figures 4A-F. K15 immunoreactivity was predominantly detected in the basal cell zone in NOM, OHK and OLP whereas in OED, basal K15 immunopositivity tends to diminish with increasing grades of dysplasia.

\section{DISCUSSION}

Although numerous studies have demonstrated the presence of stem cells in a variety of diseased and normal tissues including the pancreas, colon, lung, brain, peripheral blood and prostate ${ }^{21}$, reports on these cells in oral epithelia are limited ${ }^{13,17}$. Furthermore, accurate identification of stem cells at this tissue site is not well-established. In the oral epithelium, the putative stem/progenitor cellrelated markers for characterizing epithelial stem cells include cytokeratin (K5/14, K15, K19) $5,13,26$, integrins $(\alpha 2, \alpha 3, \alpha 6, \beta 1 \text { and } \beta 4)^{13,14}, \mathrm{NG}^{17}$ and $\mathrm{N} 1{ }^{17}$. In this study, four stem cell-like markers (integrin $\beta 1, N G 2, N 1$ and K15) were selected to examine the tissue layers of dysplastic oral epithelium for cells that expressed these proteins, taking into consideration their distribution patterns as it might reflect more accurately the effects of aberrant signalling activity on these tissues in general and on stem cells in particular. Results on their expression patterns were compared with those of OLP, non-dysplastic (OHK) and normal oral epithelium (NOM).

Integrin $\beta 1$ belongs to the large integrin family of transmembrane glycoprotein adhesion receptors that mediate cell-matrix and cell-cell adhesion. In human epidermis, integrin $\beta 1$ is an essential feature of epidermal stem cells $\mathbf{s}^{12,14,19,25}$. In the present study, among the four stem celllike protein markers evaluated, integrin $\beta 1$ was the most widely expressed in both test and control samples, being detected mainly in the basal layer. This distribution pattern corroborated a previous study in which integrin $\beta 1$ was also preferentially localized in the basal layer of NOM, OHK and OLP ${ }^{17}$. It is a generally accepted idea that the stem cell niche for oral epithelial stem cells is in the basal layer ${ }^{11}$. Another noteworthy observation is that 2 OED samples (moderate OED, $\mathrm{n}=1$; severe OED, $\mathrm{n}=1 ; 10 \%$ of total) showed evidence of suprabasal (spinous) staining for this biomarker. However, further investigation will be needed to determine the significance of this finding in relation to possible expansion of the stem cell compartment in oral dysplasia. In bovine normal stratified squamous epithelium, $\beta 1$ integrin is expressed by basal epithelial cells whereas in bovine papillomavirus-induced fibropapillomas, $\beta 1$ integrin is over-expressed and aberrantly localized in the suprabasal layer ${ }^{6}$. Given these evidences and our current observation, we speculated that a shift or expansion of the stem cell niche from the basal to the suprabasal zone in dysplastic oral epithelium may have occurred ${ }^{6,11}$.

Neuron-glial-2 (NG2) is a transmembrane proteoglycan present in oligodendrocyte progenitors ${ }^{9}$, chondroblast ${ }^{10}$ and osteoblasts ${ }^{10}$. In the present study, NG2 was generally underexpressed in both test and control specimens. Lack of immunoreactivity might be attributed to the fact that NG2 expression is mostly found in fetal and developing tissues, precisely in cells that represent an intermediate transitional stage between stem cell progenitors and fully differentiated cells such as oligodendrocyte precursor cells (OPC) in human central nervous system ${ }^{9}$. This unique transitional character of OPC may not exist in oral epithelial progenitor cells. However, NG2 was reported to be expressed outside the nervous system including skin ${ }^{15}$, as well as in many tumors such as chondrosarcomas ${ }^{18}$ and melanomas ${ }^{4}$. In this study, nearly all samples were NG2-negative. In a few cases, the observed weak NG2 positivity in the suprabasal and corneum layers differed from an earlier report ${ }^{16}$ in which NG2 was mostly expressed within the basal layer. Our present observation seems to suggest the presence of TACs in these layers rather than in the basal layer which might be colonized by stem cells. This interpretation was based on the fact that NG2 is mostly expressed by cells that have made an initial commitment to a particular cellular lineage ${ }^{9}$.

$\mathrm{K} 15$ is a marker of hair follicle stem cells ${ }^{20}$ and is also expressed in basal epidermal keratinocytes ${ }^{26}$. The positive identification of K15 in the basal layer of both normal and test samples suggests that this protein molecule is also a marker for oral epithelial stem cells. K15 localization in the cytoplasm is an expected outcome because keratins usually form intermediate filament networks that are further developed into a dense and highly dynamic cytoskeleton spanning between the nucleus and the cell membrane $e^{5,20,26}$. It is a generally accepted idea that the stem cell niche for oral epithelial stem cells is in the basal layer ${ }^{11}$. Our current observation of K15 overexpression in the basal layer, with aberrant overexpression and localization in the spinous layer of higher grades of OEDs, suggests an expansion of the stem cell niche from the basal to the suprabasal zone ${ }^{11}$. In arsenic-induced skin 
cancers, K15 increased expression was purportedly indicative of emergence of cancer stem cells ${ }^{31}$. On the other hand, another report found that poorly differentiated SCCs and SeOED are generally associated with a reduction or total loss of primary keratins $(\mathrm{K} 5, \mathrm{~K} 14 \text { and } \mathrm{K} 15)^{2}$. This reduction in expression is believed to be the cause of impaired desmosome formation with subsequent adhesion loss and increase in migratory propensity that is associated with these pathological disorders ${ }^{8}$.

Notch signaling is an evolutionarily conserved pathway that regulates numerous cellular processes including self-renewal of normal stem cells ${ }^{31}$. In mammals, there are four notch receptors. Altered notch signaling is known to affect the function of a variety of mammalian stem cells including those from marrow, skin and intestine, while deregulation of notch signaling has been implicated in tumor development and progression ${ }^{32}$. In normal squamous epithelium, N1 is predominantly expressed by basal cells but is downregulated in neoplasms of squamous origin ${ }^{23,29}$. Our current observations of weak and highly variable N1 signals in normal and dysplastic oral epithelium suggest that this protein is unreliable as a marker for epithelial stem cells.

In conclusion, this study demonstrated that subpopulations of cells with stem cell-like properties were identified in the dysplastic oral epithelium. However, these markers were unable to identify individual stem cells. The characteristic expression of K15 might be of diagnostic value for oral dysplasia and should be investigated further.

\section{ACKNOWLEDGEMENTS}

This work was funded by the University of Malaya Postgraduate Grant No. PS166/2010B and by the Ministry of Health Malaysia Fundamental Research Grant No. FP038-2013A.

\section{REFERENCES}

1- Barnes L, Eveson JW, Reichart PA, Sidransky D, eds. World Health Organization classification of tumours. Pathology and genetics of head and neck tumours. Lyon: IARC Press; 2005. 2- Bloor BK, Seddon SV, Morgan PR. Gene expression of differentiation-specific keratins in oral epithelial dysplasia and squamous cell carcinoma. Oral Oncol. 2001;37:251-61.

3- Bombeccari GP, Guzzi G, Tettamanti M, Giannì AB, Baj A, Palloti $F$, et al. Oral lichen planus and malignant transformation: a longitudinal cohort study. Oral Surg Oral Med Oral Pathol Oral Radiol Endod. 2011;112:328-34.

4- Bumol TF, Reisfeld RA. Unique glycoprotein-proteoglycan complex defined by monoclonal antibody on human melanoma cells. Proc Natl Acad Sci U S A. 1982;79:1245-9.

5- Clausen H, Vedtofte P, Moe D, Dabelsteen E, Sun TT, Dale B. Differentiation-dependent expression of keratins in human oral epithelia. J Invest Dermatol. 1986;86:249-54.

6- Cooper B, Brimer N, Stoler M, Vande Pol SB. Suprabasal overexpression of beta-1 integrin is induced by bovine papillomavirus type 1 . Viriology. 2006;355:102-14.
7- Cotsarelis G, Kaur P, Dhouailly D, Hengge U, Bickenbach J. Epithelial stem cells in the skin: definition, markers, localization and functions. Exp Dermatol. 1999;8:80-8.

8- Crowe DL, Milo GE, Shuler CF. Keratin 19 downregulation by oral squamous cell carcinoma lines increases invasive potential. J Dent Res. 1999;78:1256-63.

9- Dawson MR, Levine JM, Reynolds R. NG2-expressing cells in the central nervous system: are they oligodendroglial progenitors? J Neurosci Res. 2000;61:471-9.

10- Fukushi J, Inatani M, Yamaguchi Y, Stallcup W. Expression of NG2 proteoglycan during endochondral and intramembranous ossification. Dev Dyn. 2003;228:143-8.

11- Igarashi T, Shimmura S, Yoshida S, Tanagi M, Shinozaki N, Yamane GY. Isolation of oral epithelial progenitors using collagen IV. Oral Dis. 2008;14:413-8.

12- Janes SM, Lowell S, Hutter C. Epidermal stem cells. J Pathol. 2002;197:479-91.

13- Jones KB, Klein OD. Oral epithelial stem cells in tissue maintenance and disease: the first steps in a long journey. Int J Oral Sci. 2013;5:121-9.

14- Jones PH, Watt FM. Separation of human epidermal stem cells from transit amplifying cells on the basis of differences in integrin function and expression. Cell. 1993;73:713-24.

15- Kadoya K, Fukushi J, Matsumoto Y, Yamaguchi Y, Stallcup WB. NG2 proteoglycan expression in mouse skin: altered postnatal skin development in the NG2 null mouse. J Histochem Cytochem. 2008; 56:295-303.

16- Kelly EB. Stem cells. $1^{\text {st }}$ ed. Westport: Greenwood Publishing Group; 2007.

17- Köse O, Lalli A, Kutulola AO, Odell EW, Waseem A. Changes in the expression of stem cell markers in oral lichen planus and hyperkeratotic lesions. J Oral Sci. 2007;49:133-9.

18- Léger O, Johnson-Léger C, Jackson E, Coles B, Dean C. The chondroitin sulphate proteoglycan NG2 is a tumour-specific antigen on the chemically induced rat chondrosarcoma HSN. Int J Cancer. 1994;58:700-5.

19- Li A, Simmons PJ, Kaur P. Identification and isolation of candidate human keratinocyte stem cells based on cell surface phenotype. Proc Natl Acad Sci U S A. 1998;95:3902-7.

20- Lyle S, Christofidou-Solomidou M, Liu Y, Elder DE, Albelda $\mathrm{S}$, Cotsarelis G. The C8/144B monoclonal antibody recognizes cytokeratin 15 and defines the location of human hair follicle stem cells. J Cell Sci. 1998;111:3179-88.

21- Masters J, Bernhard O. Palsson BO. Human adult stem cells. $7^{\text {th }}$ ed. New York: Springer; 2009.

22- Morrison SJ, Kimble J. Asymmetric and symmetric stem-cell divisions in development and cancer. Nature. 2006;441:1068-74. 23- Nickoloff BJ, Qin JZ, Chaturvedi V, Denning MF, Bonish B, Miele L. Jagged-1 mediated activation of notch signaling induces complete maturation of human keratinocytes through NF-kappaB and PPARgamma. Cell Death Differ. 2002;9:842-55.

24- Pellegrini G, Dellambra E, Golisano O, Martinelli E, Fantozzi I, Bondanza S, et al. P63 identifies keratinocyte stem cells. Proc Natl Acad Sci U S A. 2001;98:3156-61.

25- Potten CS, Morris RJ. Epithelial stem cells in vivo. J Cell Sci Suppl. 1988;10:45-62.

26- Presland RB, Dale BA. Epithelial structural proteins of the skin and oral cavity: function in health and disease. Crit Rev Oral Biol Med. 2000;11:383-408.

27- Rangarajan A, Talora C, Okuyama R, Nicolas M, Mammucari $C$, Oh $\mathrm{H}$, et al. Notch signaling is a direct determinant of keratinocyte growth arrest and entry into differentiation. EMBO J. $2001 ; 20: 3427-36$.

28- Ruzzi L, Gagnoux-Palacios L, Pinola M, Belli S, Meneguzzi G, D'Alessio $M$, et al. A homozygous mutation in the integrin alpha6 gene in junctional epidermolysis bullosa with pyloric atresia. J Clin Invest.1997;99:2826-31. 
29- Sakamoto K, Fujii T, Kawachi H, Miki Y, Omura K, Morita K, et al. Reduction of NOTCH1 expression pertains to maturation abnormalities of keratinocytes in squamous neoplasms. Lab Invest. 2012;92:688-702.

30- Smith J, Rattay T, McConkey C, Helliwell T, Mehanna H. Biomarkers in dysplasia of the oral cavity: a systemic review. Oral Oncol. 2009;45:647-53.

31- Sun Y, Pi J, Wang X, Tokar EJ, Liu J, Waalkes MP. Aberrant cytokeratin expression during arsenic-induced acquired malignant phenotype in human $\mathrm{HaCaT}$ keratinocytes consistent with epidermal carcinogenesis. Toxicology. 2009;262:162-70.
32- Wang Z, Li Y, Banerjee S, Sarkar FH. Emerging role of Notch in stem cells and cancer. Cancer Lett. 2009;279:8-12.

33- Warnakulasuriya S, Johnson NW, van der Waal I. Nomenclature and classification of potentially malignant disorders of the oral mucosa. J Oral Pathol Med. 2007;36:575-8. 